\title{
Editorial
}

\section{The special issue devoted to papers from the Astrobiology Society of Britain Conference 2006}

\section{M.J. Burchell}

Centre for Astrophysics and Planetary Science, School of Physical Science, University of Kent, Canterbury, Kent CT2 7NH, UK

e-mail:m.j.burchell@kent.ac.uk

The Astrobiology Society of Britain (http://www.astrobiologysociety.org/) held its second conference (Life Here, There and Everywhere) in April 2006. The meeting was hosted by the University of Kent and took place on campus in Canterbury from 18-21 April. A total of 48 abstracts were submitted to the meeting. The final schedule featured 35 talks and 14 posters on a range of topics which included Mars, the Moon, comets, exoplanets, impacts, outreach and analytic techniques - all with an astrobiology flavour. With 62 attendees (including overseas delegates from Sweden, Spain, Portugal, Australia and the United States) the meeting also allowed for strong social interaction (and the building of new collaborations and networking) as well as showcasing ongoing research in the field.

One feature of the meeting was that several of the talks were focused on public outreach; for example, it was fascinating to hear how University researchers were interacting with eight year olds and helping them make PowerPoint talks about what aliens look like! Engaging with the public is an important part of science, not just because the work is publicly funded but also to help develop a scientific culture in our society. Astrobiology is a perfect vehicle for such outreach activities, catching the public imagination as few other sciences currently do.

Both this and the next issue of the International Journal of Astrobiology contain papers arising from the meeting. Although it was originally planned to feature the papers in just one issue of the journal, the number of high quality papers submitted exceeded the capacity of a single issue. The committee and members of the Astrobiology Society of Britain are delighted that the editors of the journal have accordingly agreed to provide space not just in this issue, but also in the next. The number, range and quality of the papers demonstrate the vitality of this research field in the UK.

It is clear that the UK astrobiology community has strengthened substantially in the last few years, with significant research work being carried out. The next conference of the society is scheduled for 2008 and by then we hope that there will be many new results to highlight. 